\title{
Derivation of the Governing Differential Equation of Vibrating Host Plate with Two Piezoelectric Patches
}

\author{
Wasim Barham \\ wsbarham@just.edu.jo \\ Civil Engineering Department, Jordan University of Science and Technology, Irbid, Jordan \\ Khaldoon Bani-Hani \\ khaldoon@just.edu.jo \\ Civil Engineering Department, Jordan University of Science and Technology, Irbid, Jordan \\ Mutaz Mohammad \\ mmmohammad16@eng.just.edu.jo \\ Civil Engineering Department, Jordan University of Science and Technology, Irbid, Jordan
}

\begin{abstract}
One of the most difficult challenges facing researchers these days is making industrial applications (e.g. engines, automobiles, and aircraft) run on renewable energy and reducing the use of fuel as much as possible. One approach to achieve this goal is the use of smart materials such as piezoelectric materials which produce mechanical stress or strain under the application of an electrical field. Vice versa, if this material is subjected to a mechanical strain, an electrical field will result. Piezoelectric materials are used as sensors or actuators for the structural control of smart structures. In this paper, a mathematical model represented by the governing differential equation for a host plate containing two piezoelectric patches has been developed to predict the displacement of a plate excited by the piezoelectric patches and transverse loads.
\end{abstract}

Keywords: Renewable energy; Piezoelectric material; Piezoelectric patches; Electric field; Host plate

\section{INTRODUCTION}

Piezoelectric materials are smart materials used in many applications such as sensor and actuator for active health monitoring (Tzou \& Tseng, 1990), aircraft morphing wing technology (Jha \& Kudva, 2004), voltage generators (Yando, 1970), and accelerometers (Scheeper et al., 1996). Piezoelectricity idiom from the Greek word "piezo" (Ueberschlag, 2001) denotes to the pressure of electricity. It is a character of some materials to produce a mechanical stress or strain on the application of an electrical field (Cheng et al., 2007), referred to as inverse piezoelectric effect, Figure 1-b. Vice versa, if this material is subjected to a mechanical strain, an electrical field will result, this is called the direct piezoelectric effect (Munjal, 2015), Figure 1-a. The direct piezoelectric effect was discovered by the two brothers Curie (1880). In 1910, Woldemar Voigt published a book named Lehrbuch der Kristallphysik which depicted many natural crystal classes that have piezoelectricity property and provided the piezoelectric constants using tensor analysis (Voigt, 1910). For a period, a lot of research was conducted to examine the crystal structure that shows piezoelectricity. The piezoelectricity can be obtained in some materials through polarization process such as ceramic, Lead Zirconate Titanate 
PZT, polyvinylidene fluoride (Ueberschlag, 2001), and Barium Titanate (Balmes E, Deraemaeker, 2013).

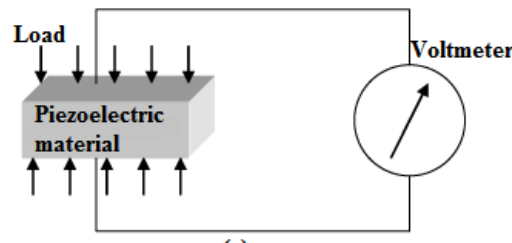

(a)

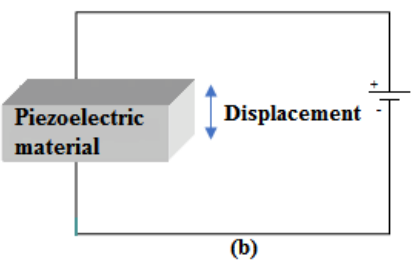

(b) Inverse piezoelectric effect

Figure 1: (a) Direct piezoelectric effect

A hysteresis of piezoelectric actuators was studied and modelled mathematically by (Singh \& Apte, 2006). Gharib et al. (2008) analyzed functionally graded beam comprising two piezoelectric patches used as sensor and actuator. Sun et al. (2001) examined the effect of controlling beams vibration with piezoelectric patches used as sensors and actuators. Energy harvesters driven by vibration effectively convert vibration energy into electrical energy using three electromechanical transduction processes: piezoelectric (Behjat et al., 2011), electrostatic (Song et al., 2016), and electromagnetic (Singh \& Apte, 2006).

In this paper, piezoelectric patches are bonded to a host plate and used as sensors and actuators. A static analysis for a piezoelectric plate has been conducted to evaluate the displacement induced by an electric field. Furthermore, mathematical model of the host plate contained piezoelectric sensor and actuator has been developed to predict the displacement of a plate excited by piezoelectric patches, subject to any function of electric field and load.

\section{PARTIAL DIFFERENTIAL EQUATION OF A PLATE WITH TWO PIEZOELECTRIC PATCHES}

In this section, the governing differential equation for the thin plate with piezoelectric sensor and actuator will be derived. Consider a rectangular plate with $a$ and $b$ dimensions and thickness $h$. The plate has piezoelectric sensor and actuator patches as shown in Figure 2 and Figure 3.



Figure 2: Rectangular plate with piezoelectric patches 


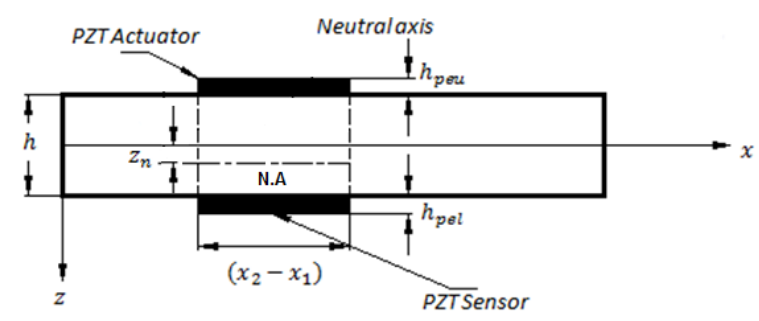

Figure 3: Elevation view

The displacement components of the host plate at a point located at a distance $z$ from the mid-surface are given by:

$$
\begin{gathered}
u=-\left(z-z_{n} S(x, y)\right) \frac{\partial w}{\partial x} \\
v=-\left(z-z_{n} S(x, y)\right) \frac{\partial w}{\partial y} \\
w=w(x, y)
\end{gathered}
$$

The displacement components of the piezoelectric patches at a point located at a distance $z$ from the mid-surface are given by:

$$
\begin{gathered}
u=-\left(z-z_{n}\right) \frac{\partial w}{\partial x} \\
v=-\left(z-z_{n}\right) \frac{\partial w}{\partial y} \\
w=w(x, y)
\end{gathered}
$$

Where $Z_{n}$ is the distance between mid-plane and neutral surface.

The shift of the neutral surface can be calculated as follows:

$$
\sum F_{x}=0
$$

Assuming that the slope of flexural stress for each material equals to the modulus of elasticity as shown in Figure 4 below:

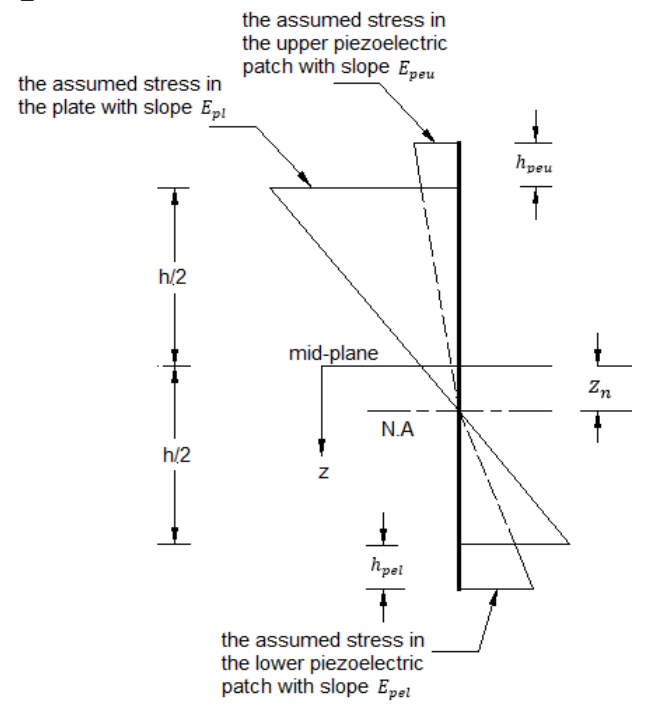

Figure 4: The assumed stress diagram to compute $\boldsymbol{Z}_{\boldsymbol{n}}$ 
One can write,

Where,

$$
\left\{\begin{array}{c}
\int_{-\left(h / 2+h_{\mathrm{peu}}\right)}^{-h / 2} E_{\mathrm{peu}}\left(z-z_{n}\right) d z \\
\int_{-h / 2}^{h / 2} E_{\mathrm{pl}}\left(z-z_{n}\right) d z \\
\int_{h / 2}^{h / 2+h_{\mathrm{pel}}} E_{\mathrm{pel}}\left(z-z_{n}\right) d z
\end{array}\right\}=0
$$

and,

$$
z_{n}=\frac{\left\{\begin{array}{c}
E_{\text {pel }} h_{\text {pel }}\left(h+h_{\text {pel }}\right) \\
-E_{\text {peu }} h_{\text {peu }}\left(h+h_{\text {puu }}\right)
\end{array}\right\}}{2\left(E_{p l} h+E_{\text {pel }} h_{p e l}+E_{\text {peu }} h_{\text {peu }}\right)}
$$

$$
S(x, y)=\left[\begin{array}{c}
H\left(x-x_{1}\right) H\left(x-x_{2}\right) \\
-H\left(x-x_{1}\right) H\left(x-x_{2}\right)
\end{array}\right]
$$

$H(x)$ is a Heaviside unit step function. For the host plate, strain functions can be written in terms of the plate deflection $w$ as follows:

$$
\begin{gathered}
\epsilon_{x}=z_{n} \frac{\partial S(x, y)}{\partial x} \frac{\partial w}{\partial x}-\left(z-z_{n} S(x, y)\right) \frac{\partial^{2} w}{\partial x^{2}} \\
\epsilon_{y}=z_{n} \frac{\partial S(x, y)}{\partial y} \frac{\partial w}{\partial y}-\left(z-z_{n} S(x, y)\right) \frac{\partial^{2} w}{\partial y^{2}} \\
\gamma_{x y}=\left\{\begin{array}{c}
\left(\frac{\partial S(x, y)}{\partial y} \frac{\partial w}{\partial x}+\frac{\partial S(x, y)}{\partial x} \frac{\partial w}{\partial y}\right) \\
-2\left(z-z_{n} S(x, y)\right) \frac{\partial^{2} w}{\partial x \partial y}
\end{array}\right\}
\end{gathered}
$$

On the other hand, for the piezoelectric patches, the strain functions can be written in terms of the plate deflection $w$ as follows:

$$
\begin{gathered}
\epsilon_{x}=-\left(z-z_{n} S(x, y)\right) \frac{\partial^{2} w}{\partial x^{2}} \\
\epsilon_{y}=-\left(z-z_{n} S(x, y)\right) \frac{\partial^{2} w}{\partial y^{2}} \\
\gamma_{x y}=-2\left(z-z_{n} S(x, y)\right) \frac{\partial^{2} w}{\partial x \partial y}
\end{gathered}
$$

Finally, the stresses in the plate and in the piezoelectric patches are respectively. 


$$
\begin{gathered}
{\left[\begin{array}{c}
\sigma_{x} \\
\sigma_{y} \\
\tau_{x y}
\end{array}\right]_{p e l}} \\
=\frac{E_{p e l}}{1-v_{p e l}^{2}}\left[\begin{array}{ccc}
1 & v_{p e l} & 0 \\
v_{p e l} & 1 & 0 \\
0 & 0 & \frac{1}{2}\left(1-v_{p e l}\right)
\end{array}\right] \\
{\left[\begin{array}{c}
-\left(z-z_{n}\right) w_{x x} \\
-\left(z-z_{n}\right) w_{y y} \\
-2\left(z-z_{n}\right) w_{x y}
\end{array}\right]-\frac{d_{31 l} E_{p e l}}{1-v_{p e l}}\left[\begin{array}{l}
1 \\
1 \\
0
\end{array}\right] E_{z l}}
\end{gathered}
$$

Where subscript 'pl', 'peu', and 'pel' indicate for plate, upper piezoelectric patch, and lower piezoelectric patch respectively. The bending and twisting moments of the plate are given by:

Where:

$$
M=M_{p l}+S(x, y)\left(M_{p e u}+M_{p e l}\right)
$$

$$
\begin{gathered}
{\left[\begin{array}{c}
M_{x} \\
M_{y} \\
M_{x y}
\end{array}\right]_{p l}^{h / 2}=\int_{-h / 2}^{h}\left[\begin{array}{c}
\sigma_{x} \\
\sigma_{y} \\
\tau_{x y}
\end{array}\right]_{p l} z d z} \\
{\left[\begin{array}{c}
M_{x} \\
M_{y} \\
M_{x y}
\end{array}\right]_{p e u}=\int_{-\left(h / 2+h_{p e u}\right)}^{-h / 2}\left[\begin{array}{c}
\sigma_{x} \\
\sigma_{y} \\
\tau_{x y}
\end{array}\right]_{p e u} z d z} \\
{\left[\begin{array}{c}
M_{x} \\
M_{y} \\
M_{x y}
\end{array}\right]_{p e l}=\int_{h / 2}^{h / 2+h_{p e l}}\left[\begin{array}{c}
\sigma_{x} \\
\sigma_{y} \\
\tau_{x y}
\end{array}\right]_{p e l} z d z}
\end{gathered}
$$

Substituting Equations (9) in Equations (11), the following expressions can be written for the bending and twisting moments in terms of the plate deflection ${ }_{w}$ :

$$
\begin{aligned}
& -D\left(w_{x x}+v_{p l} w_{y y}\right) \\
& M_{x}=-S(x, y)\left(\begin{array}{c}
\gamma_{l}-\gamma_{u}+ \\
\left(w_{x x}+v_{p e u} w_{y y}\right) \beta \\
+\left(w_{x x}+v_{p e l} w_{y y}\right) \alpha
\end{array}\right) \\
& -D\left(v_{p l} w_{x x}+w_{y y}\right) \\
& M_{y}=-S(x, y)\left(\begin{array}{c}
\gamma_{l}-\gamma_{u}+ \\
\left(v_{p e u} w_{x x}+w_{y y}\right) \beta \\
+\left(v_{p e l} w_{x x}+w_{y y}\right) \alpha
\end{array}\right) \\
& M_{x y}=\left(\begin{array}{c}
-D\left(1-v_{p l}\right) \\
-S(x, y)\left(\begin{array}{c}
\left(1-v_{\text {peu }}\right) \beta \\
+\left(1-v_{\text {pel }}\right) \alpha
\end{array}\right)
\end{array}\right) w_{\mathrm{xy}}
\end{aligned}
$$

Where $\beta, \alpha, \gamma_{u}$, and $\gamma_{l}$ are constant expressions defined as follows: 


$$
\begin{gathered}
\beta=\frac{E_{\text {peu }} h_{\text {peu }}\left(\begin{array}{c}
4 h_{\text {peu }}^{2}+6 h_{\text {peu }}\left(h+z_{n}\right) \\
+3 h\left(h+2 z_{n}\right)
\end{array}\right)}{12\left(1-v_{\text {peu }}^{2}\right)} \\
\alpha=\frac{E_{p e l} h_{p e l}\left(\begin{array}{c}
4 h_{p e l}^{2}+6 h_{p e l}\left(h-z_{n}\right) \\
+3 h\left(h-2 z_{n}\right)
\end{array}\right)}{12\left(1-v_{p e l}^{2}\right)} \\
\gamma_{u}=\frac{6 E_{\text {peu }} h_{p e u} d_{31 u}\left(h+h_{\text {peu }}\right)}{12\left(1-v_{p e u}\right)} E_{z u} \\
\gamma_{l}=\frac{6 E_{p e l} h_{p e l} d_{31 l}\left(h+h_{p e l}\right)}{12\left(1-v_{p e l}\right)} E_{z l} \\
D=\frac{E_{p l} h^{3}}{12\left(1-v_{p l}^{2}\right)}
\end{gathered}
$$

By substituting the moment expressions Equation (12) into the differential equation of equilibrium for bending of thin plate Equation (14) below:

$$
\frac{\partial^{2} M_{x}}{\partial x^{2}}+2 \frac{\partial^{2} M_{x y}}{\partial x \partial y}+\frac{\partial^{2} M_{y}}{\partial y^{2}}+q_{z}=\rho h \frac{\partial^{2} w}{\partial t^{2}}
$$

The governing differential equation in terms of the displacement ${ }_{w}$ can be expressed as:

$$
\begin{gathered}
-D \nabla^{4} w+\left(S_{x x}+S_{y y}\right)\left(\gamma_{u}-\gamma_{l}\right) \\
-\beta\left[\begin{array}{c}
S \nabla^{4} w+2 S_{x}\left(w_{\mathrm{xxx}}+w_{\mathrm{xyy}}\right) \\
+2 S_{y}\left(w_{y y y}+w_{x x y}\right) \\
+S_{x x}\left(w_{x x}+v_{p e u} w_{y y}\right) \\
+2\left(1-v_{p e u}\right) S_{x y} w_{x y} \\
+S_{y y}\left(w_{y y}+v_{p e u} w_{x x}\right)
\end{array}\right]+q_{z} \\
-\alpha\left[\begin{array}{c}
S \nabla^{4} w+2 S_{x}\left(w_{\mathrm{xxx}}+w_{\mathrm{xyy}}\right) \\
+2 S_{y}\left(w_{y y y}+w_{x x y}\right) \\
+S_{x x}\left(w_{x x}+v_{p e l} w_{y y}\right) \\
+2\left(1-v_{p e l}\right) S_{x y} w_{x y}+ \\
S_{y y}\left(w_{y y}+v_{p e l} w_{x x}\right)
\end{array}\right] \\
=\rho h \frac{\partial w^{2}}{\partial t^{2}}
\end{gathered}
$$

It is worth mentioning here that the first term represents the effect of the host plate, the second term represents the effect of the electric field difference between the two patches, and the third and the fourth terms account for the effect of the upper and lower piezoelectric patches' properties respectively. Various methods for analysis of plates 
were developed for arbitrary shapes. However, in many cases, the exact solution cannot be obtained, therefore, numerical methods such as finite element method, finite divided difference, and Galerkin method can be used to get approximate solution.

\section{CONCLUSION}

An analytical model for a host plate with two piezoelectric patches under the condition of free vibration has been developed and the governed partial differential equation has been derived. The model can be applied to a rectangular plate with any boundary conditions. One of the most important features of this model is that the neutral axis for the host plate is represented by Heaviside step function since it shifted by $z_{n}$ at patches location. It can also be concluded that if the electric field is zero in both patches then the governing partial differential equation (15) becomes identical to Kirchhoff equation with the effect of material properties of the patches included.

\section{REFERENCES}

Balmes E. \& Deraemaeker A. (2013). Modeling structures with piezoelectric materials. SDT tutorial.

Behjat B., Salehi M., Armin A., Sadighi M. \& Abbasi M. (2011). Static and dynamic analysis of functionally graded piezoelectric plates under mechanical and electrical loading. Scientia Iranica, 18(4):986-94.

Cheng L., Zhang H. \& Li Q. (2007). Design of a capacitive flexible weighing sensor for vehicle WIM system. Sensors. 7(8):1530-44.

Curie J. (1880). Développement par compression de l'électricité polaire dans les cristaux hémièdres à faces inclinées. Bull Soc Fr Mineral, 3:90.

Gharib A., Salehi M. \& Fazeli S. (2008). Deflection control of functionally graded material beams with bonded piezoelectric sensors and actuators. Materials Science and Engineering, A. 498(1-2):110-4.

Jha A. K. \& Kudva J. N. (2004). Morphing aircraft concepts, classifications, and challenges. Smart Structures and Materials: Industrial and Commercial Applications of Smart Structures Technologies, International Society for Optics and Photonics.

Munjal B. S. (2015). Finite element analysis design development of smart skin spacecraft antenna reflectors using intelligent smart materials structural systems, Gujarat University.

Scheeper P., Gulløv J. O. \& Kofoed L. M. (1996). A piezoelectric triaxial accelerometer. Journal of Micromechanics and Microengineering, 6(1):131.

Singh A. \& Apte D. (2006). Modelling and analysis of hysteresis in piezoelectric actuator. Defence Science Journal, 56(5):825.

Song Y., Yang C. H., Hong S. K., Hwang S. J., Kim J. H., Choi J. Y., et al. (2016). Road energy harvester designed as a macro-power source using the piezoelectric effect. International Journal of Hydrogen Energy, 41(29):12563-8.

Sun D., Tong L. \& Atluri S. N. (2001). Effects of piezoelectric sensor/actuator debonding on vibration control of smart beams. International Journal of Solids and Structures, 38(50- 
51):9033-51.

Tzou H. \& Tseng C. (1990). Distributed piezoelectric sensor/actuator design for dynamic measurement/control of distributed parameter systems: a piezoelectric finite element approach. Journal of sound and vibration, 138(1):17-34.

Ueberschlag P. (2001). PVDF piezoelectric polymer. Sensor review, 21(2):118-26.

Voigt W. (1910). Lehrbuch der kristallphysik: BG Terebner. Leipzig, 964.

Yando S. (1970). Piezoelectric voltage generator. Google Patents. 